\title{
Dynamics of a Gross-Pitaevskii Equation with Phenomenological Damping
}

\author{
Renato Colucci, Gerardo R. Chacón, and Andrés Vargas \\ Departamento de Matemáticas, Pontificia Universidad Javeriana, Carrera 7 No. 40-62, Bogotá, Colombia \\ Correspondence should be addressed to Andrés Vargas; a.v4rg4s@gmail.com
}

Received 19 April 2013; Accepted 3 June 2013

Academic Editor: Norio Yoshida

Copyright (C) 2013 Renato Colucci et al. This is an open access article distributed under the Creative Commons Attribution License, which permits unrestricted use, distribution, and reproduction in any medium, provided the original work is properly cited.

\begin{abstract}
We study the dynamical behavior of solutions of an $n$-dimensional nonlinear Schrödinger equation with potential and linear derivative terms under the presence of phenomenological damping. This equation is a general version of the dissipative GrossPitaevskii equation including terms with first-order derivatives in the spatial coordinates which allow for rotational contributions. We obtain conditions for the existence of a global attractor and find bounds for its dimension.
\end{abstract}

\section{Introduction}

Nonlinear Schrödinger equations have enjoyed a considerable amount of attention during several decades due to their frequent appearance in the modeling of interesting physical phenomena in many different areas (e.g., optics, fluid mechanics, condensed matter, etc.). Additionally, the rigorous mathematical treatment of these equations has produced a great deal of new insights and techniques accompanied by a voluminous bibliography. We refer the reader to excellent sources as [1-4] for a detailed account of these aspects.

Here, for complex coefficients $\alpha$ and $\beta$, our purpose is to study the long-term dynamics of a general nonlinear Schrödinger evolution equation with potential of the form

$$
i u_{t}-\alpha \Delta u+\beta|u|^{2} u+f(x) u=v(x) \cdot \nabla u,
$$

where $u(x, t)$ and $f(x)$ are complex-valued functions and $v(x)$ is a complex vector-valued map, all defined on a bounded open domain $U \subset \mathbb{R}^{n}$ (for $n \geqslant 1$ ) with regular boundary $\partial U$. We prove the existence of a global attractor for (1), under Dirichlet and $U$-periodic boundary conditions, assuming $\operatorname{Im}(\alpha)>0$ and some additional requirements on $f(x)$ and $v(x)$ for which existence and uniqueness of solutions are guaranteed. Our choice of signs for the terms in this equation was made such that the hypotheses needed for our analysis make, if necessary, the imaginary parts of constant and functional coefficients positive.
Particular instances of (1) have been extensively considered in the literature, specially in two and three dimensions. In fact, when the coefficients $\alpha$, and $\beta$ and the function $f(x)$ are real and assuming $v(x)=0$, this is the celebrated time-dependent Gross-Pitaevskii equation (GP) employed to model nonlinear behavior in several physical systems. Of special relevance is the mean-field description of BoseEinstein condensation achieved in dilute atomic gases, a state of matter which exhibits peculiar phenomena characteristic of the superfluid nature of the system.

If for the previous situation in three dimensions we choose $v(x)=i \Omega \wedge x \in i \mathbb{R}^{3}$, where $\Omega \in \mathbb{R}^{3}$ is an angular velocity vector, we recover the GP equation with angular momentum rotational term. Indeed, the right-hand side of (1) becomes

$$
v \cdot \nabla u=i(\Omega \wedge x) \cdot \nabla u=i \Omega \cdot(x \wedge \nabla) u=-\Omega \cdot L u,
$$

where $L:=-i(x \wedge \nabla)$ is precisely the quantum mechanical angular momentum operator. This equation has been used to model physical experiments where the condensate is set into rotation with an angular frequency $\|\Omega\|$, making it known as the Gross-Pitaevskii equation for rotating Bose gases. In this situation, the observation of quantized vortices has been a topic of considerable experimental and theoretical studies; the reader is referred to [5] and references therein for details on vortices in Bose-Einstein condensates and the recent review [6] for a treatment that includes numerical aspects 
(see also [7] for the simulation of rotating condensates). Existence and uniqueness results for solutions of GP equation with rotation in $\mathbb{R}^{n}$ for dimensions $n=2$ and $n=3$ and under different assumptions can be found in [8-10]. Note that rotating and nonnecessarily nonlinear Schrödinger equations appear also in several other contexts related to wave propagation (see references in [11]).

The general case of fully complex coefficients $\alpha, \beta$ and complex-valued functions $f(x), v(x)$ in (1) can be thought of as a mixture between the aforementioned GP equation and a gradient flow which accounts for dissipation in the system proportional to the rate of change of the wave function $u(x, t)$. This is indeed the case if (1) has the particular form

$$
i u_{t}=(1+i \gamma)\left[\alpha \Delta u-\beta|u|^{2} u-f(x) u+v(x) \cdot \nabla u\right],
$$

for real constants $\alpha, \beta, \gamma$, a real-valued function $f(x)$, and an imaginary vector field $v(x)$ as in the rotational term explained earlier. In the physical literature phenomenological damping has been introduced to study Bose-Einstein condensates (both in the rotating and nonrotating cases) affected by complicated sources of dissipation related to their interaction with the thermal cloud around the condensate. For example, [12] studies the case of trapped atomic condensates and [13] analyzes dark matter wave solitons in the same context, while [14-16] study the formation of vortex lattices in rotating condensates, including numerical simulations. A theoretical explanation of the phenomenological damping terms can be found in [17] (see also [18, 19]).

From the mathematical side, equations similar to (3), but with $v=0$, have been used to study the behavior of GinzburgLandau vortices in [20] and, in the case of low dissipation, the asymptotics for perturbation of constant solutions in [21]. Long-time behavior, establishing the existence of attractors under several assumptions, has also been the topic of several studies; see, for example, [22] for Ginzburg-Landau equations on bounded domains, [23] for weakly damped nonlinear Schrödinger equations in $\mathbb{R}^{n}$ with $n \leqslant 3$, and for dissipative nonlinearities see [24] and references therein. To our knowledge attractors for the dissipative Gross-Pitaevskii equation with rotation have not been explicitly considered.

Since the arguments needed for our analysis work in the fully complex coefficients situation, we adopt this broader setting and, in addition, do not necessarily assume that the right-hand side of (1) has the particular form of an angular momentum contribution. This situation corresponds to the case where the ratio between imaginary and real parts of $\alpha$, $\beta, f(x)$, and $v(x)$ in (3) is not a fixed constant $\gamma$. In fact, this can include cases where there are sources of dissipation which are proportional to some of the individual terms in the equation, like the nonlinearity or the gradient terms. Such contributions are important, for example, to study exploding and collapsing Bose-Einstein condensates (see [25] and references therein for examples of pure nonlinear dissipation in that context, as well as [26] for simulation of collapse and explosion).

We point out that numerical studies in [27] show that cubic or quintic damping terms prevent blowup of solutions to focusing nonlinear Schrödinger equations without "rotational" term, while just linear damping is not enough below a certain threshold. This agrees with Theorem 8 on existence of attractors (that covers such situation if one assumes an arbitrarily small amount of linear damping) and provides some justification for the hypotheses under which this result is proved.

Finally, to summarize the content of the following sections we mention that, under appropriate assumptions introduced later, Theorem 4 establishes the existence and uniqueness of solutions to the dissipative GP equation on bounded open domains, Theorem 8 shows the existence of a compact global attractor, and Theorem 11 provides bounds for its Hausdorff and fractal dimensions.

1.1. Precise Setting. To ease notation, for any complex vector $v \in \mathbb{C}^{n}$ with $n \geqslant 1$, we define $v_{1}:=\operatorname{Re}(v) \in \mathbb{R}^{n}$ and $v_{2}:=$ $\operatorname{Im}(v) \in \mathbb{R}^{n}$. Let $U \subset \mathbb{R}^{n}$; if $u(x, t)=u_{1}(x, t)+i u_{2}(x, t)$ : $U \times \mathbb{R}^{+} \rightarrow \mathbb{C}$ is a complex-valued function, the gradient $\nabla u$ and Laplacian $\Delta u$ (both with respect to spatial coordinates) are given by $\nabla u:=\nabla u_{1}+i \nabla u_{2} \in \mathbb{C}^{n}$ and $\Delta u:=\Delta u_{1}+i \Delta u_{2} \in \mathbb{C}$. In addition, we extend bilinearly the Euclidean inner product “." in $\mathbb{R}^{n}$ to a symmetric inner product on $\mathbb{C}^{n}$ denoted for simplicity by the same dot; that is, for vectors $v, w \in \mathbb{C}^{n}$, we have

$$
v \cdot w:=\left(v_{1} \cdot w_{1}-v_{2} \cdot w_{2}\right)+i\left(v_{1} \cdot w_{2}+v_{2} \cdot w_{1}\right) .
$$

With this product, the standard Hermitian norm of $v \in \mathbb{C}^{n}$ satisfies $\|v\|^{2}=\left\|v_{1}\right\|^{2}+\left\|v_{2}\right\|^{2}=v \cdot \bar{v}$, where $\bar{v}:=v_{1}-i v_{2}$.

Given coefficients $\alpha, \beta \in \mathbb{C}$ and a constant $p \geqslant 2$, we consider the following dissipative (fully complex) GP equation with nonlinearity of $\operatorname{order}(p+1)$ on a bounded open domain $U \subset \mathbb{R}^{n}$, for arbitrary $n \geqslant 1$ :

$$
\begin{gathered}
i u_{t}-\alpha \Delta u+\beta|u|^{p} u+f(x) u=v(x) \cdot \nabla u, \\
u(x, 0)=u_{0}(x)
\end{gathered}
$$

with unknown $u(x, t): U \times \mathbb{R}^{+} \rightarrow \mathbb{C}$, a scalar function $f(x)$ : $U \rightarrow \mathbb{C}$, and a vector-valued map $v(x): U \rightarrow \mathbb{C}^{n}$. Our hypotheses on the constant coefficients are

$$
\begin{gathered}
\alpha_{2}>0, \\
\beta_{2} \geqslant p\left|\beta_{1}\right| \geqslant 0
\end{gathered}
$$

and on the functional coefficients and initial data are

$$
\begin{gathered}
f(x) \in L^{\infty}(U), \\
v(x) \in L^{\infty}\left(U, \mathbb{C}^{n}\right), \\
u_{0}(x) \in L^{2}(U) .
\end{gathered}
$$

Additionally, we assume that

$$
\mu_{2}:=\underset{x \in U}{\operatorname{ess} \inf _{0}} f_{2}(x)>0
$$

and require that

$$
4 \alpha_{2} \mu_{2}>\|v\|_{\infty}^{2} .
$$


Remark 1. Notice that hypothesis (H2) allows for $\beta=0$ in which case there is no nonlinearity term in (5). Independently, hypotheses (H1) and (H6) already imply (H7) when $v(x)=0$ and this includes the standard GP equation (i.e., no rotational term) but with dissipation.

Remark 2. As a consequence of hypothesis (H6), (5) has a nonzero linear damping term of the form if $f_{2}(x) u$. Obviously, such term is always present in the case of GP with phenomenological damping (3) and is not the only source of dissipation.

For (5) we will consider Dirichlet boundary conditions on the domain $U \subset \mathbb{R}^{n}$, which is assumed to have regular boundary $\partial U$. Explicitly, we suppose that for all $t \geqslant 0$,

$$
\begin{gathered}
\left.u(x, t)\right|_{\partial U}=0, \\
\left.\Delta u(x, t)\right|_{\partial U}=0 .
\end{gathered}
$$

Alternatively we also consider $U$-periodic boundary conditions when the domain has the form $U=(0, L)^{n} \subset \mathbb{R}^{n}$. This means that for any pair of points $a, b \in \bar{U}=[0, L]^{n}$ which are equal except at an arbitrary fixed component $j \in\{1, \ldots, n\}$ for which $a_{j}=0$ and $b_{j}=L$, it holds that

$$
\partial^{k} u(a, t)=\partial^{k} u(b, t)
$$

for all $t \geqslant 0$ and admissible integers $k \geqslant 0$. In this expression, $\partial^{0} u:=u$ and $\partial^{k} u$ denotes any $k$-order partial (weak) derivative admissible for $u$.

Remark 3. In the case of $U$-periodic boundary conditions, it is actually assumed that points of the form $a, b \in[0, L]^{n}$ described earlier are identified, so in fact any function defined on $U$ must take the same values on those points but, in contrast to Dirichlet conditions, the precise value does not have to be fixed.

Existence and uniqueness of solutions to (5) with Dirichlet (D1)-(D2) or $U$-periodic (P) boundary conditions hold from classical results.

Theorem 4. Given hypotheses $(H 1)-(H 7)$, under Dirichlet or $U$-periodic boundary conditions, there exists a unique solution $u$ of (5) such that

$$
u \in \mathscr{C}_{b}\left(\mathbb{R}^{+}, H^{1}(U)\right), \quad u_{t} \in \mathscr{C}_{b}\left(\mathbb{R}^{+}, H^{-1}(U)\right)
$$

where $H^{-1}$ is the dual space of $H^{1}$ and $\mathscr{C}_{b}\left(\mathbb{R}^{+}, H^{1}(U)\right)$ is the set of continuous bounded functions from $\mathbb{R}^{+}$into $H^{1}(U)$.

The proof of the previous theorem follows by application of the standard Faedo-Galerkin method with small modifications to the proof of [28, Theorems IV.5.1 and IV.6.1]. The necessary estimates are similar to the ones given later to show the existence of an absorbing set in $L^{2}(U)$. It is important to notice that the estimates use only the $L^{2}$-norm of $u_{0}$, which exists by (H5).

\section{Existence of a Global Attractor}

In this section we prove the existence of a compact global attractor $\mathscr{A} \subset L^{2}(U)$. That is, a compact subset of $L^{2}(U)$ such that if $S(t)$ denotes the semigroup generated by solutions of (5), then

(i) $S(t) \mathscr{A}=\mathscr{A}$ for all $t \geqslant 0$;

(ii) for every bounded set $B \subset L^{2}(U)$, it holds that the $L^{2}$ distance $d(S(t) B, \mathscr{A}) \rightarrow 0$ as $t \rightarrow \infty$.

In order to prove the existence of the attractor, we will show first the existence of an absorbing set in $L^{2}(U)$, that is, a set $\mathscr{B}$ such that any bounded set of $L^{2}(U)$ enters into $\mathscr{B}$ after a certain time.

Theorem 5. Assume that hypotheses (H1)-(H7) hold for (5) under Dirichlet or $U$-periodic boundary conditions. Then there exist positive real constants $\tau_{0}$ and $\tau_{1}$ such that

(i) the semigroup $S(t)$ possesses an absorbing ball in $L^{2}(U)$ as

$$
\left\|S(t) u_{0}\right\|_{L^{2}} \leqslant r_{0} \quad \text { if } t \geqslant \tau_{0},
$$

where $r_{0}:=1$ and $\tau_{0}:=(1 / K) \log \left\|u_{0}\right\|_{L^{2}}$, with $K:=$ $\mu_{2}-\left(1 / 4 \alpha_{2}\right)\|v\|_{\infty}^{2}$;

(ii) the semigroup $S(t)$ possesses an absorbing ball in $H_{0}^{1}(U)$ as

$$
\left\|S(t) u_{0}\right\|_{H_{0}^{1}} \leqslant r_{1} \quad \text { if } t \geqslant \tau_{1} \text {, }
$$

$$
\text { where } r_{1}:=\left(1 / \alpha_{2}\right) e^{(1 / 2)\|v\|_{\infty}^{2}}\left(\|v\|_{\infty}^{2}+\alpha_{2}^{2}\|f\|_{\infty}^{2}+1\right)^{1 / 2}
$$
and $\tau_{1}:=\tau_{0}+\alpha_{2}$.

From the previous theorem we have that the set

$$
\mathscr{B}:=\left\{u \in L^{2}(U):\|u\|_{L^{2}} \leqslant r_{0},\|\nabla u\|_{L^{2}} \leqslant r_{1}\right\} \subset H^{1}(U)
$$

is absorbing for all the bounded sets of $L^{2}(U)$ provided that $t \geqslant \tau_{1}$.

Proof. We divide the proof into two steps. Before that, we recall the following inequality for the convenience of the reader (see [28, Lemma III.1.1] for a proof).

Lemma 6 (uniform Gronwall's lemma [29]). Let $f$, $g$, and $h$ be locally integrable functions on $\left(t_{0}, \infty\right)$ such that $f^{\prime}$ is locally integrable on $\left(t_{0}, \infty\right)$ and $f^{\prime} \leqslant f g+h$, for all $t \geqslant t_{0}$. Assume additionally that there are real positive constants $a_{1}, a_{2}, a_{3}$ such that for every $t \geqslant t_{0}$,

$$
\begin{gathered}
\int_{t}^{t+\varrho} f(s) d s \leqslant a_{1}, \quad \int_{t}^{t+\varrho} g(s) d s \leqslant a_{2}, \\
\int_{t}^{t+\varrho} h(s) d s \leqslant a_{3} .
\end{gathered}
$$

Then it holds that

$$
f(t+\varrho) \leqslant\left(\frac{a_{1}}{\varrho}+a_{3}\right) e^{a_{2}}, \quad \forall t \geqslant t_{0} .
$$


2.1. Absorbing Ball in $L^{2}(U)$. Multiplying (5) by $\bar{u}$, integrating over $U \subset \mathbb{R}^{n}$ using integration by parts with any set of the proposed boundary conditions, and taking the imaginary part,

$$
\begin{aligned}
& \frac{1}{2} \frac{d}{d t}\|u\|_{L^{2}}^{2}+\alpha_{2}\|\nabla u\|_{L^{2}}^{2}+\beta_{2}\|u\|_{L^{p+2}}^{p+2}+\int_{U} f_{2}(x)|u|^{2} d x \\
& \quad=\operatorname{Im}\left(\int_{U}(v \cdot \nabla u) \bar{u} d x\right) \\
& \quad \leqslant\|v \cdot \nabla u\|_{L^{2}}\|u\|_{L^{2}} \\
& \quad \leqslant\|\nabla u\|_{L^{2}}\|v\|_{\infty}\|u\|_{L^{2}} \\
& \quad \leqslant \alpha_{2}\|\nabla u\|_{L^{2}}^{2}+\frac{1}{4 \alpha_{2}}\|v\|_{\infty}^{2}\|u\|_{L^{2}}^{2} .
\end{aligned}
$$

After eliminating the third term to the left-hand side of the previous calculation, we get

$$
\frac{d}{d t}\|u\|_{L^{2}}^{2} \leqslant-2 K\|u\|_{L^{2}}^{2}
$$

where

$$
K=K\left(\alpha_{2}, f_{2}, v\right):=\mu_{2}-\frac{1}{4 \alpha_{2}}\|v\|_{\infty}^{2}
$$

Then, applying Gronwall's lemma and since (H7) implies that $K>0$, we obtain

$$
\|u\|_{L^{2}}^{2} \leqslant\left\|u_{0}\right\|_{L^{2}}^{2} e^{-2 K t}
$$

Setting $\tau_{0}:=(1 / K) \log \left\|u_{0}\right\|_{L^{2}}$, we have that

$$
\|u(t)\|_{L^{2}} \leqslant 1=: r_{0}, \quad \text { if } t \geqslant \tau_{0} .
$$

This proves the existence of an absorbing ball in $L^{2}(U)$.

2.2. Absorbing Ball in $H_{0}^{1}(U)$. Now we multiply (5) by $\Delta \bar{u}$, integrate as before over $U \subset \mathbb{R}^{n}$ using boundary conditions (after integration by parts) to simplify, and take the imaginary part to get

$$
\begin{aligned}
\frac{1}{2} \frac{d}{d t}\|\nabla u\|_{L^{2}}^{2}+\alpha_{2}\|\Delta u\|_{L^{2}}^{2}+\operatorname{Im}\left(\int_{U}(v \cdot \nabla u) \Delta \bar{u} d x\right) \\
=\operatorname{Im}\left(\int_{U} f(x) u \Delta \bar{u} d x\right)+\operatorname{Im}\left(\beta \int_{U}|u|^{p} u \Delta \bar{u} d x\right) .
\end{aligned}
$$

Integrating by parts the last term of the previous equality and using that for any $z, w \in \mathbb{C}$ it holds that $\operatorname{Im}(z w)=z_{1} w_{2}+$ $z_{2} w_{1}$, we get

$$
\begin{aligned}
& \operatorname{Im}\left(\beta \int_{U}|u|^{p} u \Delta \bar{u} d x\right) \\
&=-\beta_{2} \int_{U}|u|^{p}\|\nabla u\|^{2} d x \\
&-p \operatorname{Im}\left(\beta \int_{U}|u|^{p-2}(u \nabla \bar{u}) \cdot \operatorname{Re}(u \nabla \bar{u}) d x\right) \\
&=-\beta_{2} \int_{U}|u|^{p}\|\nabla u\|^{2} d x \\
&-p \beta_{1} \int_{U}|u|^{p-2} \operatorname{Im}(u \nabla \bar{u}) \cdot \operatorname{Re}(u \nabla \bar{u}) d x \\
&-p \beta_{2} \int_{U}|u|^{p-2}\|\operatorname{Re}(u \nabla \bar{u})\|^{2} d x \\
& \leqslant-\beta_{2} \int_{U}|u|^{p}\|\nabla u\|^{2} d x \\
&+p\left|\beta_{1}\right| \int_{U}|u|^{p-2}\|u \nabla \bar{u}\|^{2} d x \\
& \leqslant-\left(\beta_{2}-p\left|\beta_{1}\right|\right) \int_{U}|u|^{p}\|\nabla u\|^{2} d x,
\end{aligned}
$$

where the inequality appears after eliminating the last term since $\beta_{2} \geqslant 0$. Replacing this in the original equation, we can write

$$
\begin{aligned}
\frac{1}{2} \frac{d}{d t}\|\nabla u\|_{L^{2}}^{2}+\alpha_{2}\|\Delta u\|_{L^{2}}^{2}+\left(\beta_{2}-p\left|\beta_{1}\right|\right) \int_{U}|u|^{p}\|\nabla u\|^{2} d x \\
\leqslant \operatorname{Im}\left(\int_{U} f(x) u \Delta \bar{u} d x\right)-\operatorname{Im}\left(\int_{U}(v \cdot \nabla u) \Delta \bar{u} d x\right) \\
\leqslant\|f\|_{\infty}\|u\|_{L^{2}}\|\Delta u\|_{L^{2}}+\|v\|_{\infty}\|\nabla u\|_{L^{2}}\|\Delta u\|_{L^{2}} \\
\leqslant \frac{1}{2 \alpha_{2}}\|f\|_{\infty}^{2}\|u\|_{L^{2}}^{2}+\frac{\alpha_{2}}{2}\|\Delta u\|_{L^{2}}^{2} \\
+\frac{1}{2 \alpha_{2}}\|v\|_{\infty}^{2}\|\nabla u\|_{L^{2}}^{2}+\frac{\alpha_{2}}{2}\|\Delta u\|_{L^{2}}^{2} .
\end{aligned}
$$

Simplifying, using $\beta_{2} \geqslant p\left|\beta_{1}\right|$ and working with $t \geqslant \tau_{0}$ so that $\|u\|_{L^{2}} \leqslant r_{0}$, we arrive at

$$
\frac{d}{d t}\|\nabla u\|_{L^{2}}^{2} \leqslant \frac{1}{\alpha_{2}}\left(r_{0}^{2}\|f\|_{\infty}^{2}+\|v\|_{\infty}^{2}\|\nabla u\|_{L^{2}}^{2}\right) .
$$

To apply the uniform Gronwall lemma, it is necessary to have an upper bound on $\int_{t}^{t+\varrho}\|\nabla u\|_{L^{2}}^{2} d s$. From (12), we have

$$
\begin{aligned}
& \frac{1}{2} \frac{d}{d t}\|u\|_{L^{2}}^{2}+\alpha_{2}\|\nabla u\|_{L^{2}}^{2}+\beta_{2}\|u\|_{L^{p+2}}^{p+2}+\int_{U} f_{2}(x)|u|^{2} d x \\
& \leqslant\|\nabla u\|_{L^{2}}\|v\|_{\infty}\|u\|_{L^{2}} \\
& \quad \leqslant \frac{\alpha_{2}}{2}\|\nabla u\|_{L^{2}}^{2}+\frac{1}{2 \alpha_{2}}\|v\|_{\infty}^{2}\|u\|_{L^{2}}^{2} .
\end{aligned}
$$


Now, eliminating the last two positive terms at the left-hand side, using $t \geqslant \tau_{0}$ again, and integrating, we find that

$$
\int_{t}^{t+\varrho}\|\nabla u\|_{L^{2}}^{2} d s \leqslant r_{0}^{2}\left(\frac{\varrho}{\alpha_{2}^{2}}\|v\|_{\infty}^{2}+\frac{1}{\alpha_{2}}\right)=: a_{1} .
$$

There are also obvious bounds given by

$$
\begin{gathered}
\int_{t}^{t+\varrho} \frac{1}{\alpha_{2}}\|v\|_{\infty}^{2} d s \leqslant \frac{\varrho}{\alpha_{2}}\|v\|_{\infty}^{2}=: a_{2}, \\
\int_{t}^{t+\varrho} \frac{1}{\alpha_{2}} r_{0}^{2}\|f\|_{\infty}^{2} d s \leqslant \frac{\varrho}{\alpha_{2}} r_{0}^{2}\|f\|_{\infty}^{2}=: a_{3} .
\end{gathered}
$$

With this, Lemma 6 immediately implies

$$
\|\nabla u(t+\varrho)\|_{L^{2}}^{2} \leqslant r_{0}^{2}\left(\frac{1}{\alpha_{2}^{2}}\|v\|_{\infty}^{2}+\frac{1}{\alpha_{2} \varrho}+\frac{\varrho}{\alpha_{2}}\|f\|_{\infty}^{2}\right) e^{\left(\varrho / \alpha_{2}\right)\|v\|_{\infty}^{2}} .
$$

Choosing $\varrho=\alpha_{2}$ and assuming $t \geqslant \tau_{1}:=\tau_{0}+\alpha_{2}$, the previous inequality becomes

$$
\|\nabla u(t)\|_{L^{2}} \leqslant \frac{r_{0}}{\alpha_{2}} e^{(1 / 2)\|v\|_{\infty}^{2}}\left(1+\|v\|_{\infty}^{2}+\alpha_{2}^{2}\|f\|_{\infty}^{2}\right)^{1 / 2}=: r_{1} .
$$

This proves the existence of an absorbing ball in $H_{0}^{1}(U)$.

To show the existence of a compact global attractor in $L^{2}(U)$, we will make use of the following result (see [28, Theorem I.1.1]) with $H=L^{2}(U)$.

Theorem 7. Suppose that $S(t)$ is a continuous semigroup acting on a metric space $\mathscr{H}$ such that there exists a bounded absorbing set $\mathscr{B}$ and for every bounded set $B \subset \mathscr{H}$ there exists $t_{0}>0$ such that $\bigcup_{t \geqslant t_{0}} S(t) B$ is relatively compact in $\mathscr{H}$. Then the $\omega$-limit set

$$
\omega(\mathscr{B}):=\bigcap_{s \geqslant 0} \overline{\bigcup_{t \geqslant s} S(t) \mathscr{B}}
$$

is a compact global attractor for the semigroup $S(t)$ in $\mathscr{H}$.

Theorem 8. Under hypotheses (H1)-(H7) for (5), supplemented with Dirichlet or U-periodic boundary conditions, there exists a compact global attractor $\mathscr{A} \subset L^{2}(U)$.

Proof. We will make use of Theorem 7. Since we have already shown the existence of the inertial set, we only need to prove that for any bounded set $B \in L^{2}(U)$, there exists $t_{0}>0$ such that the set $\bigcup_{t \geqslant t} S(t) B$ is relatively compact in $L^{2}(U)$. As a consequence of Theorem 5 , we have proved the existence of a ball

$$
\mathscr{B}:=\left\{u \in L^{2}(U):\|u\|_{L^{2}} \leqslant r_{0},\|\nabla u\|_{L^{2}} \leqslant r_{1}\right\} \subset H^{1}(U),
$$

such that for any bounded set $B \subset L^{2}(U)$, it holds that $S(t) B \subset \mathscr{B}$ for $t \geqslant \tau_{1}$. Since $\mathscr{B}$ is bounded in $H^{1}(U)$ and the embedding of $H^{1}(U)$ in $L^{2}(U)$ is compact, we conclude that the set

$$
\bigcup_{t \geqslant \tau_{1}} S(t) B
$$

is relatively compact in $L^{2}(U)$. This finishes the proof.

Remark 9. We point out here that when no dissipation is assumed in (5), which requires in particular $\alpha_{2}=\beta_{2}=0$, $f_{2}(x)=0$, and $v_{1}(x)=0$, a very different situation may occur. For example, for the nondissipative Gross-Pitaevskii equation with rotation, it has been proved in [10, Theorem 2.2] that there are conditions on $f_{1}(x)$ and $v_{2}(x)$ such that (5) has initial data $u_{0}(x)$ for which solutions present finite time blowup in dimensions $n=2$ or $n=3$; that is, there is a finite time $\tau_{*}>0$ such that

$$
\lim _{t \rightarrow \tau_{*}}\|\nabla u(t)\|_{L^{2}}=\infty
$$

This can happen, for example, with an axial-symmetric parabolic potential for $n=2,3$ in the case of a focusing $\left(\beta_{1}>0\right)$ and energy subcritical nonlinearity of appropriate order (more precisely $4 / n \leqslant p<4 /(n-2)$ with $p$ even). In our situation, the hypotheses employed (in particular the kind of dissipation) eliminate the possibility of such phenomena.

\section{Dimension of the Attractor}

In this section we provide an estimate for the dimension of the attractor by the method of evolution of infinitesimal $m$-dimensional volume elements in the phase space $L^{2}(U)$ under the flow induced by the action of the semigroup $S(t)$ of Theorem 5 (see [28, Chapter V] for details). For this estimate we will explicitly assume that the bounded open domain $U \subset$ $\mathbb{R}^{n}$ is at least of class $\mathscr{C}^{1}$; that is, $U$ has a boundary $\partial U$ with a structure of an $(n-1)$-dimensional manifold of class $\mathscr{C}^{1}$.

Given an orthonormal basis $\varphi_{1}(t), \ldots, \varphi_{m}(t)$ for a timedependent $m$-dimensional subspace of $L^{2}(U)$, it is possible (cf. [28, Appendix, Corollary 4.1]) to find constants $c_{1}, c_{2} \geqslant 0$, depending on $n, k$ and the shape of $U \subset \mathbb{R}^{n}$ but independent of the size $|U|$, the value $m$, and of the chosen orthonormal basis, such that

$$
\sum_{i=1}^{m}\left\|\nabla \varphi_{i}\right\|_{L^{2}}^{2} \geqslant \frac{1}{|U|^{2 / n}}\left(c_{1} m^{1+2 / n}-c_{2} m\right)
$$

Moreover, if the functions $\varphi_{i} \in H_{0}^{1}(U)$, it is possible to choose $c_{2}=0$. This holds for Dirichlet boundary conditions.

In order to study the evolution of infinitesimal volume elements, we linearize (5) around a solution $u(t)=S(t) u_{0}$, with $u_{0} \in \mathscr{A}$ as follows:

$$
\begin{gathered}
i \phi_{t}=F^{\prime}(u) \phi:=\alpha \Delta \phi-\beta(p+1)|u|^{p} \phi-f(x) \phi+v \cdot \nabla \phi, \\
\phi(0)=\xi .
\end{gathered}
$$


Then, noting that $\operatorname{Re}\left(\phi_{t}\right)=\operatorname{Im}\left(F^{\prime}(u) \phi\right)$, the evolution of the $m$-dimensional volume elements is given by (cf. [30, Section 13.2])

$$
V_{m}(t)=V_{m}(0) \exp \left[\operatorname{Im}\left(\int_{0}^{t} \operatorname{Tr}\left(F^{\prime}(u) P_{m}(s)\right) d s\right)\right],
$$

where $P_{m}$ is the orthogonal projection of $L^{2}(U)$ over the subspace spanned by the first $m$ eigenvectors of the Laplacian. The asymptotic growth rate of $V_{m}(t)$ is given by

$$
\lim _{t \rightarrow \infty} \frac{1}{t} \int_{0}^{t} \operatorname{Im}\left[\operatorname{Tr}\left(F^{\prime}(u) P_{m}(s)\right)\right] d s .
$$

Since we need the maximal growth rate, we compute the supremum over all $u_{0} \in \mathscr{A}$ and over all the $m$-dimensional projectors as follows:

$$
\sup _{u_{0} \in \mathscr{A}} \sup _{P_{m}} \limsup _{t \rightarrow+\infty} \frac{1}{t} \int_{0}^{t} \operatorname{Im}\left[\operatorname{Tr}\left(F^{\prime}(u) P_{m}(s)\right)\right] d s=: \operatorname{Tr}_{m}(\mathscr{A}) \text {. }
$$

In order to have exponential decay, we look for the smallest $m$ such that the number $\operatorname{Tr}_{m}(\mathscr{A})$ is negative. In detail, let $\varphi_{1}(t), \ldots, \varphi_{m}(t)$ be an orthonormal basis for $P_{m} L^{2}(U)$; then

$$
\operatorname{Im}\left[\operatorname{Tr}\left(F^{\prime}(u) P_{m}\right)\right]=\sum_{i=1}^{m} \operatorname{Im}\left[\left\langle F^{\prime}(u) \varphi_{i}, \varphi_{i}\right\rangle_{L^{2}(U)}\right],
$$

with

$$
\begin{aligned}
\operatorname{Im}\left[\left\langle F^{\prime}(u) \varphi_{i}, \varphi_{i}\right\rangle_{L^{2}(U)}\right] & \\
= & -\alpha_{2}\left\|\nabla \varphi_{i}\right\|_{L^{2}}^{2}-\beta_{2}(p+1) \int_{U}|u|^{p}\left|\varphi_{i}\right|^{2} d x \\
& -\int_{U} f_{2}(x)\left|\varphi_{i}\right|^{2} d x+\operatorname{Im}\left(\int_{U}\left(v \cdot \nabla \varphi_{i}\right) \bar{\varphi}_{i} d x\right) .
\end{aligned}
$$

Now, we estimate each of the last three terms separately. For the first, since $\beta_{2} \geqslant 0$, we can simply write

$$
-\beta_{2}(p+1) \int_{U}|u|^{p}\left|\varphi_{i}\right|^{2} d x \leqslant 0,
$$

for the second, we have

$$
-\int_{U} f_{2}(x)\left|\varphi_{i}\right|^{2} d x \leqslant-\mu_{2}\left\|\varphi_{i}\right\|_{L^{2}}^{2}=-\mu_{2}<0,
$$

and for the last term,

$$
\begin{aligned}
\operatorname{Im} \int_{U}\left(v \cdot \nabla \varphi_{i}\right) \bar{\varphi}_{i} d x & \leqslant \int_{U}\left|v \cdot \nabla \varphi_{i}\right|\left|\varphi_{i}\right| d x \\
& \leqslant \int_{U}\|v\|\left\|\nabla \varphi_{i}\right\|\left|\varphi_{i}\right| d x \\
& \leqslant\|v\|_{\infty} \int_{U}\left\|\nabla \varphi_{i}\right\|\left|\varphi_{i}\right| d x \\
& \leqslant\|v\|_{\infty}\left\|\nabla \varphi_{i}\right\|_{L^{2}}\left\|\varphi_{i}\right\|_{L^{2}} \\
& \leqslant \frac{\alpha_{2}}{2}\left\|\nabla \varphi_{i}\right\|_{L^{2}}^{2}+\frac{\|v\|_{\infty}^{2}}{2 \alpha_{2}} .
\end{aligned}
$$

Replacing these bounds in (35), we get for the imaginary part of the trace

$$
\begin{aligned}
& \operatorname{Im}\left[\operatorname{Tr}\left(F^{\prime}(u) P_{m}\right)\right] \\
& \quad \leqslant-\frac{\alpha_{2}}{2} \sum_{i=1}^{m}\left\|\nabla \varphi_{i}\right\|_{L^{2}}^{2}-m \mu_{2}+m \frac{\|v\|_{\infty}^{2}}{2 \alpha_{2}} \\
& \quad \leqslant-\frac{\alpha_{2} c_{1}}{2|U|^{2 / n}} m^{1+2 / n}+m\left(\frac{\alpha_{2} c_{2}}{2|U|^{2 / n}}+\frac{\|v\|_{\infty}^{2}}{2 \alpha_{2}}-\mu_{2}\right) \\
& \leqslant-\kappa_{1} m^{1+2 / n}+\kappa_{2},
\end{aligned}
$$

where we have used (30) and a Young inequality to obtain the required constants $\kappa_{i}$ given by

$$
\begin{gathered}
\kappa_{1}:=\left(\frac{\alpha_{2} c_{1}}{n+2}\right)|U|^{-2 / n} \\
\kappa_{2}:=\frac{2|U|^{-1}}{(n+2)}\left(\frac{\alpha_{2} c_{1}}{2}\right)^{n / 2}\left(\frac{\alpha_{2} c_{2}}{2|U|^{2 / n}}+\frac{\|v\|_{\infty}^{2}}{2 \alpha_{2}}-\mu_{2}\right)^{1+n / 2} .
\end{gathered}
$$

Remark 10. Notice, however, that in (40) the use of a Young inequality requires

$$
\mu_{2}<\frac{\alpha_{2} c_{2}}{2|U|^{2 / n}}+\frac{\|v\|_{\infty}^{2}}{2 \alpha_{2}}
$$

which produces a well-defined positive real constant $\kappa_{2}$. In particular, for Dirichlet boundary conditions (where $c_{2}$ can be taken to be zero), in addition to hypothesis (H7), this requirement reads $\|v\|_{\infty}>2 \alpha_{2} \mu_{2}$. It is also possible to replace $\mu_{2}$ by zero in the expression for $\kappa_{2}$ and the bound is still fine but not as sharp. See Remark 12 if (42) does not hold.

This provides an estimate for the dimension of the attractor $\mathscr{A}$. In fact, similarly to what is done in $[28$, Section VI.7] using Proposition V.2.1 and Theorem V.3.3 there (see also [30, Theorem 2.4]), we have the following theorem.

Theorem 11. Under the assumptions of Theorem 8 and if (42) holds, let $m \in \mathbb{N}$ be such that

$$
m-1<\left(\frac{2 \kappa_{2}}{\kappa_{1}}\right)^{n /(n+2)} \leqslant m
$$

Then the infinitesimal $m$-dimensional volume elements in phase space $L^{2}(U)$ decay exponentially as $t \rightarrow \infty$. Moreover, the global attractor $\mathscr{A}$ has finite Hausdorff dimension $\operatorname{dim}_{H}(\mathscr{A}) \leqslant m$ and finite fractal dimension $\operatorname{dim}_{F}(\mathscr{A}) \leqslant 2 m$. 
Explicitly, we have that

$$
m \sim 2^{n /(n+2)}\left[\left(\frac{\alpha_{2} c_{1}}{4|U|}\right)^{(n-2) /(n+2)}\left(\frac{\alpha_{2} c_{2}}{2|U|^{2 / n}}+\frac{\|v\|_{\infty}^{2}}{2 \alpha_{2}}-\mu_{2}\right)\right]^{n / 2} .
$$

Remark 12. Actually, a bound on the Hausdorff dimension is already obtained by finding the minimum value of $m \in \mathbb{N}$ in $(40)$, such that $\operatorname{Im}\left[\operatorname{Tr}\left(F^{\prime}(u) P_{m}\right)\right]<0$ (see aforementioned references). Therefore, $\operatorname{dim}_{H}(\mathscr{A})$ is strictly smaller than (44) without the $2^{n /(n+2)}$ factor. This also shows that if (42) does not hold, the Hausdorff dimension is strictly less than 1.

As a final conclusion, we stress the fact that the vector coefficient $v(x)$ of the "rotational" term does indeed increase the dimension of the attractor in a factor roughly proportional to $\|v\|_{\infty}^{n}$, where $n$ is the dimension of the ambient space. This should be compared with the role played by this term in proving finite time blowup for the nondissipative focusing case in [10], alluded in Remark 9.

\section{Acknowledgments}

The authors are indebted to the referee for indicating relevant references concerning numerical studies of Bose-Einstein condensates. This work was partially supported by Research Project "Dynamics of a nonlinear Schrödinger equation", ID-PROY 004850 of the Faculty of Sciences of Pontificia Universidad Javeriana, Bogotá, Colombia.

\section{References}

[1] J. Bourgain, Global Solutions of Nonlinear Schrödinger Equations, vol. 46 of American Mathematical Society Colloquium Publications, American Mathematical Society, Providence, RI, USA, 1999.

[2] R. Carles, Semi-Classical Analysis for Nonlinear Schrödinger Equations, World Scientific, Hackensack, NJ, USA, 2008.

[3] T. Tao, Nonlinear Dispersive Equations, vol. 106 of CBMS Regional Conference Series in Mathematics, Published for the Conference Board of the Mathematical Sciences, Washington, DC, USA, 2006.

[4] F. Linares and G. Ponce, Introduction to Nonlinear Dispersive Equations, Universitext, Springer, New York, NY, USA, 2009.

[5] A. Aftalion, Vortices in Bose-Einstein Condensates, vol. 67 of Progress in Nonlinear Differential Equations and their Applications, Birkhäuser, Boston, Mass, USA, 2006.

[6] W. Bao and Y. Cai, "Mathematical theory and numerical methods for Bose-Einstein condensation," Kinetic and Related Models, vol. 6, no. 1, pp. 1-135, 2013.

[7] W. Bao, Q. Du, and Y. Zhang, "Dynamics of rotating BoseEinstein condensates and its efficient and accurate numerical computation," SIAM Journal on Applied Mathematics, vol. 66, no. 3, pp. 758-786, 2006.

[8] C. Hao, L. Hsiao, and H.-L. Li, "Global well posedness for the Gross-Pitaevskii equation with an angular momentum rotational term," Mathematical Methods in the Applied Sciences, vol. 31, no. 6, pp. 655-664, 2008.
[9] C. Hao, L. Hsiao, and H.-L. Li, "Global well posedness for the Gross-Pitaevskii equation with an angular momentum rotational term in three dimensions," Journal of Mathematical Physics, vol. 48, no. 10, Article ID 102105, 11 pages, 2007.

[10] P. Antonelli, D. Marahrens, and C. Sparber, "On the Cauchy problem for nonlinear Schrödinger equations with rotation," Discrete and Continuous Dynamical Systems A, vol. 32, no. 3, pp. 703-715, 2012.

[11] H. Liu, "Critical thresholds in the semiclassical limit of 2-D rotational Schrödinger equations," Zeitschrift für Angewandte Mathematik und Physik, vol. 57, no. 1, pp. 42-58, 2006.

[12] S. Choi, S. A. Morgan, and K. Burnett, "Phenomenological damping in trapped atomic Bose-Einstein condensates," Physical Review A, vol. 57, no. 5, pp. 4057-4060, 1998.

[13] P. G. Kevrekidis and D. J. Frantzeskakis, "Multiple dark solitons in Bose-Einstein condensates at finite temperatures," Discrete and Continuous Dynamical Systems S, vol. 4, no. 5, pp. 1199-1212, 2011.

[14] M. Tsubota, K. Kasamatsu, and M. Ueda, "Vortex lattice formation in a rotating Bose-Einstein condensate," Physical Review A, vol. 65, no. 2, Article ID 023603, 2002.

[15] K. Kasamatsu, M. MacHida, N. Sasa, and M. Tsubota, “Threedimensional dynamics of vortex-lattice formation in Bose-Einstein condensates," Physical Review A, vol. 71, no. 6, Article ID 063616, 2005.

[16] L. H. Wen and X. B. Luo, "Formation and structure of vortex lattices in a rotating double-well Bose-Einstein condensate," Laser Physics Letters, vol. 9, no. 8, pp. 618-624, 2012.

[17] C. W. Gardiner, J. R. Anglin, and T. I. A. Fudge, "The stochastic Gross-Pitaevskii equation," Journal of Physics B, vol. 35, no. 6, pp. 1555-1582, 2002.

[18] C. W. Gardiner and M. J. Davis, "The stochastic Gross-Pitaevskii equation: II," Journal of Physics B, vol. 36, no. 23, pp. 4731-4753, 2003.

[19] A. S. Bradley and C. W. Gardiner, "The stochastic Gross-Pitaevskii equation III," http://arxiv.org/abs/cond-mat/0602162.

[20] M. Kurzke, C. Melcher, R. Moser, and D. Spirn, "Dynamics for Ginzburg-Landau vortices under a mixed flow," Indiana University Mathematics Journal, vol. 58, no. 6, pp. 2597-2622, 2009.

[21] E. Miot, "Damped wave dynamics for a complex GinzburgLandau equation with low dissipation," http://128.84.158.119/ abs/1003.5375v1.

[22] J.-M. Ghidaglia and B. Héron, "Dimension of the attractors associated to the Ginzburg-Landau partial differential equation," Physica D, vol. 28, no. 3, pp. 282-304, 1987.

[23] P. Laurençot, "Long-time behaviour for weakly damped driven nonlinear Schrödinger equations in $\mathbb{R}^{N}, N \leq 3$," NoDEA. Nonlinear Differential Equations and Applications, vol. 2, no. 3, pp. 357-369, 1995.

[24] N. Kita and A. Shimomura, "Large time behavior of solutions to Schrödinger equations with a dissipative nonlinearity for arbitrarily large initial data," Journal of the Mathematical Society of Japan, vol. 61, no. 1, pp. 39-64, 2009.

[25] P. Antonelli and C. Sparber, "Global well-posedness for cubic NLS with nonlinear damping," Communications in Partial Differential Equations, vol. 35, no. 12, pp. 2310-2328, 2010.

[26] W. Bao, D. Jaksch, and P. A. Markowich, "Three-dimensional simulation of jet formation in collapsing condensates," Journal of Physics B, vol. 37, no. 2, pp. 329-343, 2004.

[27] W. Bao and D. Jaksch, "An explicit unconditionally stable numerical method for solving damped nonlinear Schrödinger 
equations with a focusing nonlinearity," SIAM Journal on Numerical Analysis, vol. 41, no. 4, pp. 1406-1426, 2003.

[28] R. Temam, Infinite-Dimensional Dynamical Systems in Mechanics and Physics, vol. 68 of Applied Mathematical Sciences, Springer, New York, NY, USA, 2nd edition, 1997.

[29] C. Foiaş and G. Prodi, "Sur le comportement global des solutions non-stationnaires des équations de Navier-Stokes en dimension 2," Rendiconti del Seminario Matematico della Università di Padova, vol. 39, pp. 1-34, 1967.

[30] J. C. Robinson, Infinite-Dimensional Dynamical Systems. An Introduction to Dissipative Parabolic PDEs and the Theory of Global Attractors, Cambridge Texts in Applied Mathematics, Cambridge University Press, Cambridge, Mass, USA, 2001. 


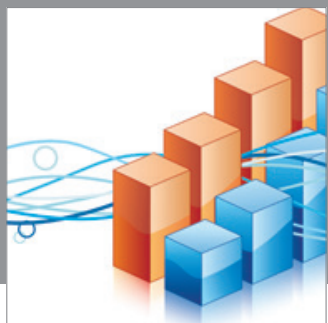

Advances in

Operations Research

mansans

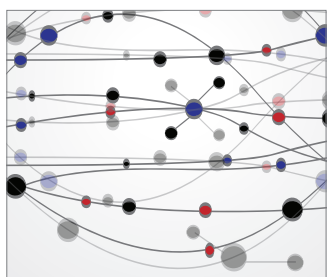

The Scientific World Journal
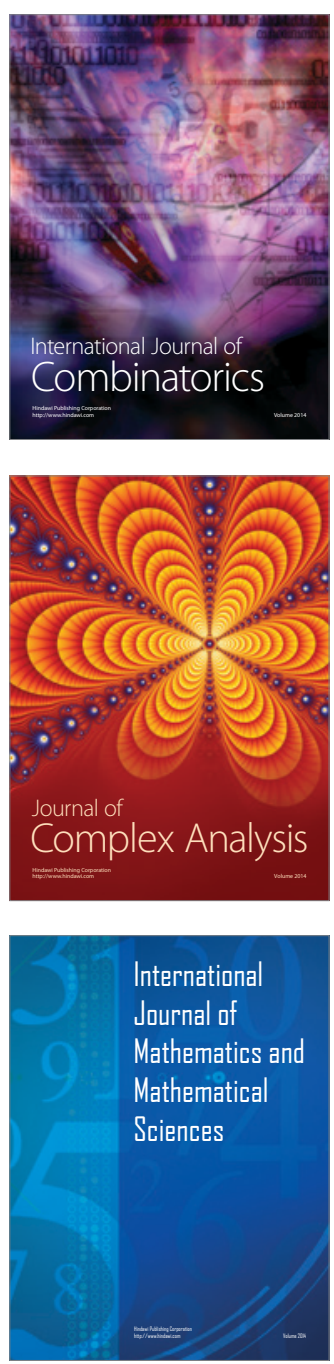
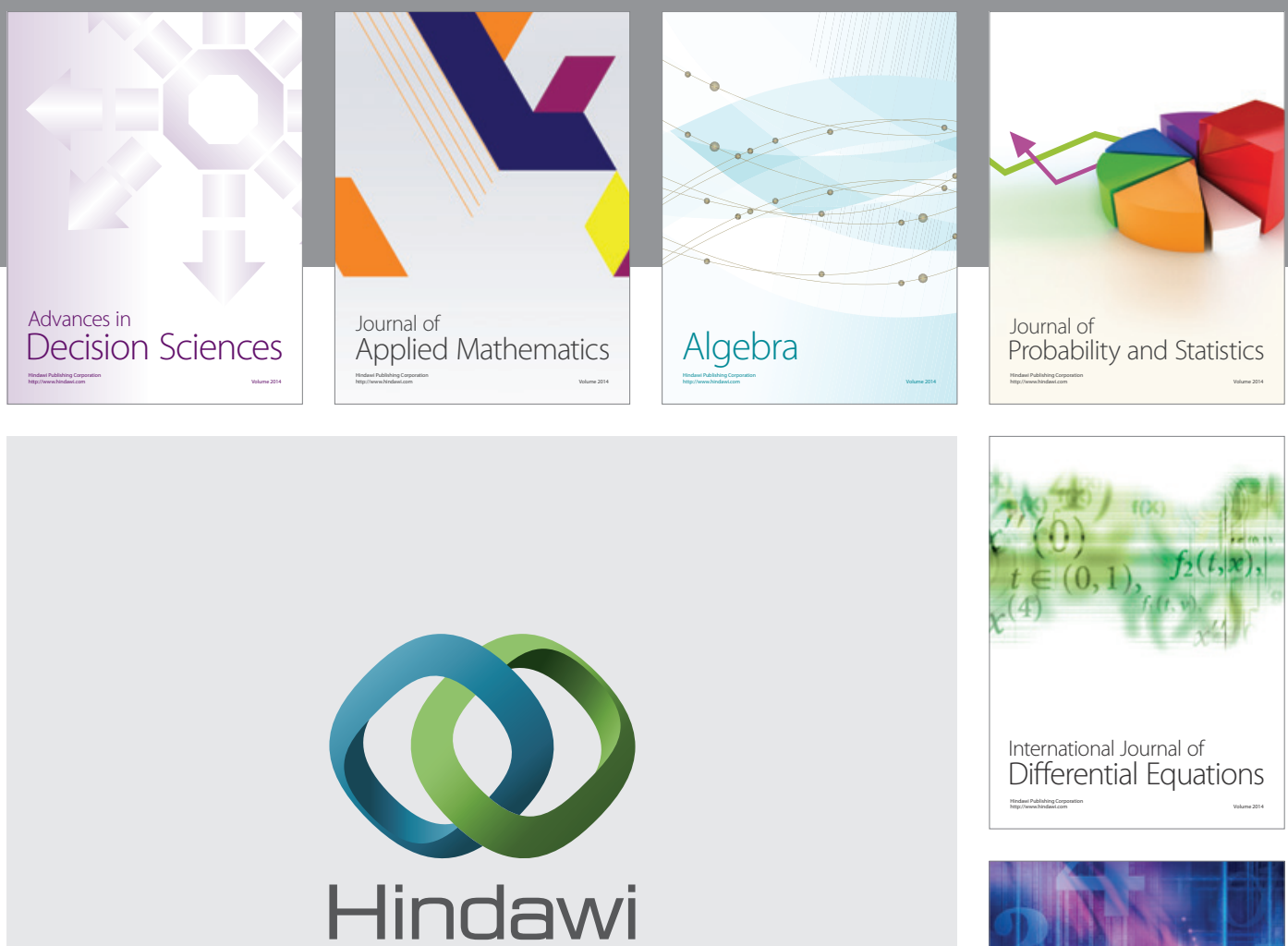

Submit your manuscripts at http://www.hindawi.com
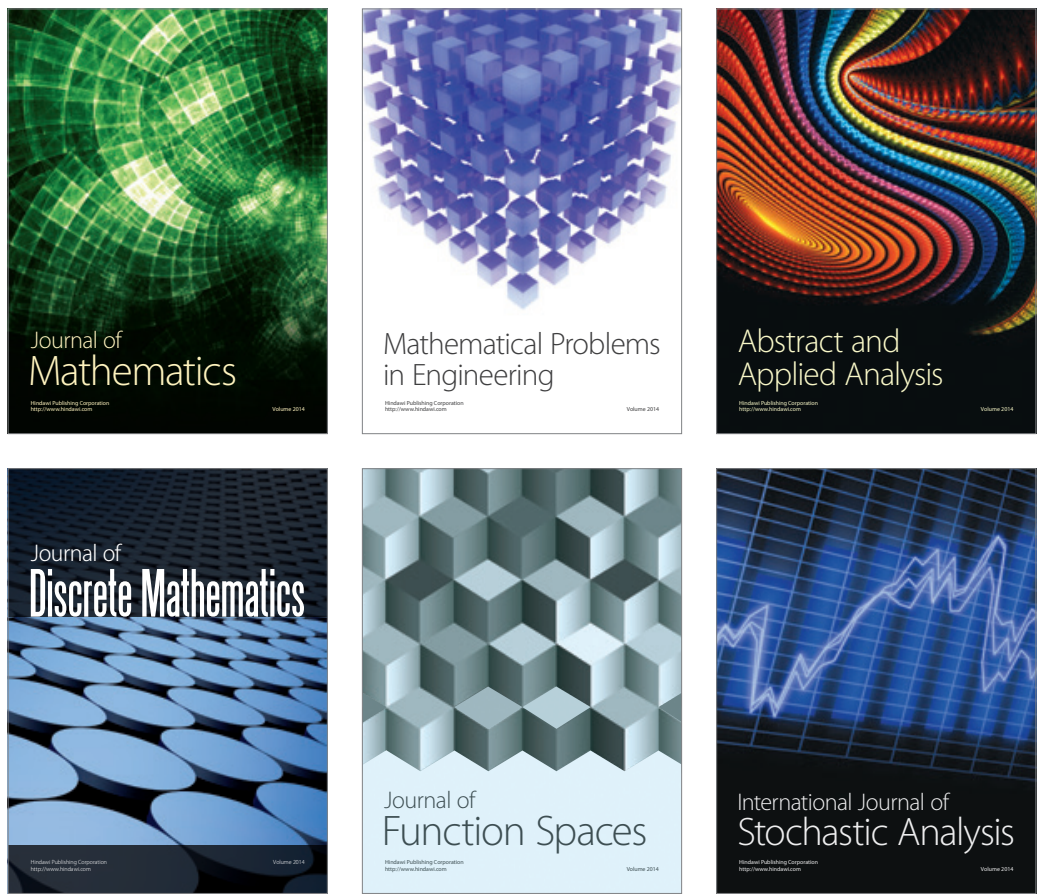

Journal of

Function Spaces

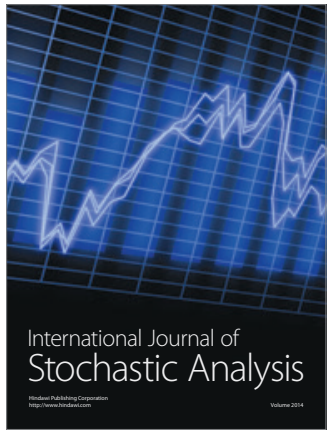

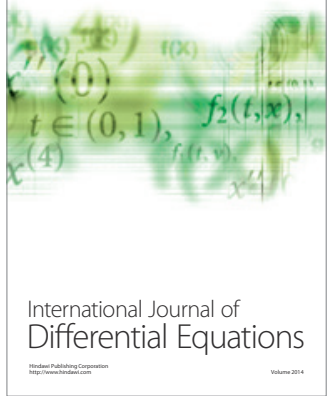
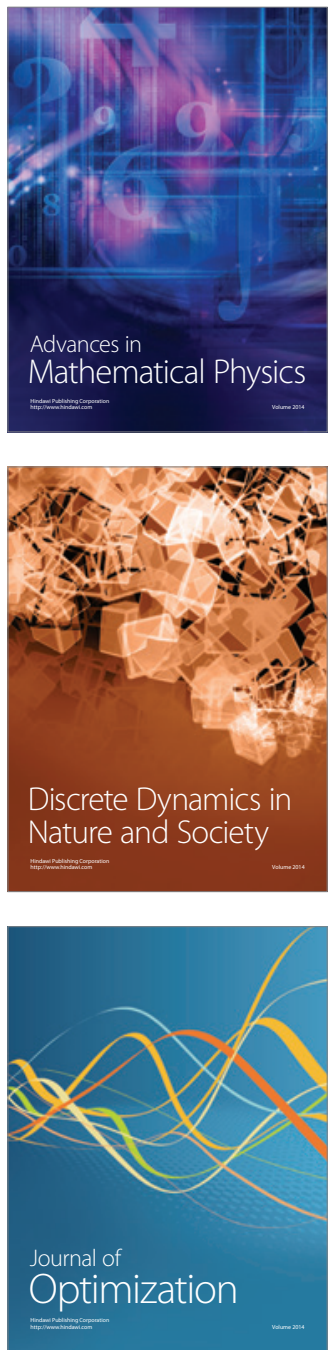